\title{
Semiring Pruning for Information Dissemination in Mobile Ad Hoc Networks
}

\author{
Kiran K. Somasundaram, John S. Baras \\ Institute of Systems Research and Department of Electrical and Computer Engineering \\ University of Maryland, College Park \\ College Park, Maryland 20742 \\ Email: kirans@umd.edu,baras@isr.umd.edu
}

\begin{abstract}
Link state routing mechanisms have shown good convergence behaviour in networks with mobile hosts. Compared to traditional link state mechanisms, which suffer from broadcast storm problems in Mobile Ad Hoc Networks (MANETs), pruned link state approaches such as those used in Optimized Link State Routing (OLSR) have shown significant reduction in the control overhead. In this paper, we show that the pruning function is a fundamental component of link state routing protocols for MANETs, and develop a class of pruning methods for many commonly used routing objectives. We show that the ability of our local pruning methods to preserve optimal paths is a special case of the semiring distribution property.
\end{abstract}

\section{Introduction}

Routing in MANETs has been a subject of significant research over the past decade. It is common to classify MANET routing protocols as distance vector and link state routing protocols. Though architecturally different, both mechanisms compute a route profile (a route might be a path or a set of paths to reach a destination set) by optimizing the same cost function. However, the classification arises from the type of information available for the minimization and the role of minimizing/routing agents. In this paper, we relate these properties to the functional description of the Selector of Topology Information to Disseminate Component (STIDC), which was introduced in the component-based architecture for MANET routing protocols ([10]). We develop instances of the STIDC that guarantee desired global properties for the routing. We show that the ability of our algorithms to preserve certain properties globally by localized pruning is a manifestation of a general theory of semiring distribution.
This paper is organized as follows. In section 1, we introduce the mathematical notations. In sections 3 and 4 , we present the architectural difference between distance vector and link state mechanisms. In section 5, we summarize the component architecture of link state routing protocols. In section 6 , we detail the functioning of the STIDC and present different realizations for the STIDC. Finally in section 7, we generalize these realizations using an ordered semiring algebra.

\section{Mathematical Notation}

Let $G(V, E[t]), t \geq 0$, denote a dynamic graph, where the vertex set $V$ represents the mobile stations, and the dynamic edge set $E[t]$ represents the adjacency between a pair of stations at time $t$. Stations $i, j \in V$ are adjacent at time $t$ iff $(i, j) \in E[t]$. In this paper, we consider only undirected links/edges. Such links are typically established using neighbor discovery mechanisms, similar to those described in [3]. The one-hop neighborhood boundary of station $i, \partial \mathcal{N}_{1}^{b}(i)[t]$, is the set of nodes that have a direct adjacency to $i$. The nodes that share an adjacency with the nodes in $\partial \mathcal{N}_{1}^{b}(i)[t]$ but not with $i$ form the two-hop neighborhood boundary, which is denoted by $\partial \mathcal{N}_{2}^{b}(i)[t]$. Similarly, the r-hop neighborhood boundary, $\partial \mathcal{N}_{r}^{b}(i)[t]$, is the set of nodes that share an adjacency with $\partial \mathcal{N}_{r-1}^{b}(i)[t]$ but not with $\partial \mathcal{N}_{j}^{b}(i)[t], j<r-1$ and $i$. The neighbor discovery mechanism at station $i \in V$ typically makes visible, the $k$-hop neighborhood, $\mathcal{N}_{k}(i)[t]=\{i\} \cup_{j \leq k} \partial \mathcal{N}_{j}^{b}(i)[t]$, and the link metric weights for each edge in the subgraph of $G[t]$ induced by the vertex subset $\mathcal{N}_{k}(i)[t]$. We denote the network diameter at time $t$ by $D_{N W}[t]$.

\section{Link State Routing Protocols}

Link state routing protocols have a significant importance in the history of routing in data networks. A notable one was in the stabilization of the ARPANET 
routing protocol. The original routing protocol proposed for the wired ARPANET was an adaptive shortest path routing scheme [5]. At every router $i \in V$, the length of the link to router $j \in V$ was chosen to be the delay seen at the interface to $j$. The shortest path delay computations were based on the BellmanFord equation [5]. Every station computed the shortest path to reach a destination set by message passing in their local neighborhood, $\mathcal{N}_{1}(i)$. However, this routing mechanism was not able to cope up with the delay dynamics at the interface queues, and hence, the routing paths exhibited oscillations. After a decade of modifications and improvements, a new routing paradigm for the ARPANET was introduced in [7]. The modified new routing protocol is similar to modern link state protocols such as OSPF. The protocol involved local delay averaging (filtering) for every 10 seconds and network-wide broadcasting of the delay states every 60 seconds [5]. In this case, since the link state (delay) information is available at every router $i \in V$, the routers can locally compute the Bellman-Ford equations. This modification showed better stability properties for delay-aware routing in ARPANET [8]. This property of link state routing protocols made them attractive for dynamic networks, which also encompass mobile networks. For instance, preliminary studies by Johnson [4] show that link state routing protocols exhibit better convergence properties for networks with mobile hosts. This improved stability is due to the ability of link state algorithms to process/filter local information, which is elaborated in the forthcoming section.

\section{Network Neighborhood Computation}

In the context of routing, every station can be considered as a routing agent attempting to minimize a global cost function. In the case of distance vector mechanisms, the agents perform a local minimization, and exchange this processed information. On the contrary, in pure link state mechanisms the nodes broadcast raw (unprocessed) information. This raw information creates a complete global view of the network information for each routing agent to autonomously minimize the global cost. We observe that there are two fundamental network operations involved in these mechanisms :

1) Neighborhood processing - Processing the local raw information to prune the search space for the global minimization.

2) Network broadcasting - Broadcasting the processed information to all the routing agents to perform the global optimization.
This is illustrated in Fig. 1. While pure link state mechanisms have no neighborhood-based processing, the distance vector methods perform only neighborhoodbased processing and no broadcasting. This suggests that it is meaningful to classify routing protocols based on the neighborhood over which the network processing is carried out. This classification is illustrated in Fig. 2. It shows that pure link state algorithms, which perform no neighborhood-based computation (network processing), broadcast a lot of information. The pure distance vector approach performs $D_{N W}$ (network diameter) wide network processing, and hence, does not broadcast any raw information. The same figure also shows algorithms such as OLSR ([14], [11]), which have access to a local view of $\mathcal{N}_{2}(i), i \in V$, perform local network processing to reduce the broadcast information. In a similar manner, mechanisms that have access to $\mathcal{N}_{k}(i), i \in V$, can significantly reduce the broadcast information at the cost of local network processing. In the coming sections, we visit the component architecture proposed in [10], and relate the neighborhood-based computation to the functional description of the STIDC.

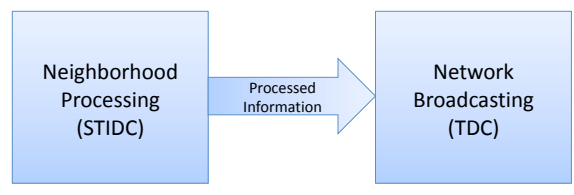

Figure 1: Fundamental components for information dissemination in routing protocols.

\section{Component Architecture for Link State Routing Algorithms}

The fundamental idea of our previous work was to identify and partition the primary functionalities of routing protocols into components. To illustrate this idea, let us consider the different functionalities of the OLSR protocol. The OLSR neighbor discovery mechanism enables every station to be aware of $\mathcal{N}_{2}(i), i \in V$. This is captured by the Neighbor Discovery Component (NDC). OLSR's MPR selection, which is based on a local vertex covering problem [2], serves two purposes: (i) choosing the subset of topology information that must be broadcast; (ii) nominating the stations to relay this information. These two functionalities can be logically partitioned into the Selector of Topology Information to Disseminate Component 


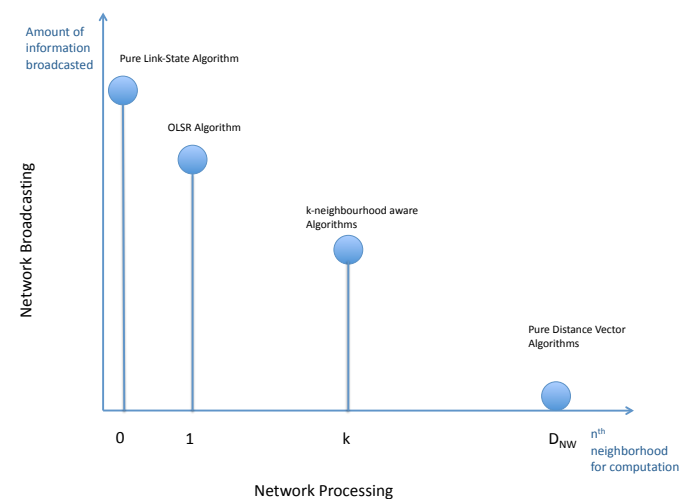

Figure 2: Relative contribution of the fundamental components.

(STIDC) and the Topology Dissemination Component (TDC) respectively. These components feed into the Route Selection Component (RSC), which builds the routing tables. These components are shown in Fig. 3. In this paper, we define the functional requirements of the STIDC, and provide a design mechanism to meet these requirements.

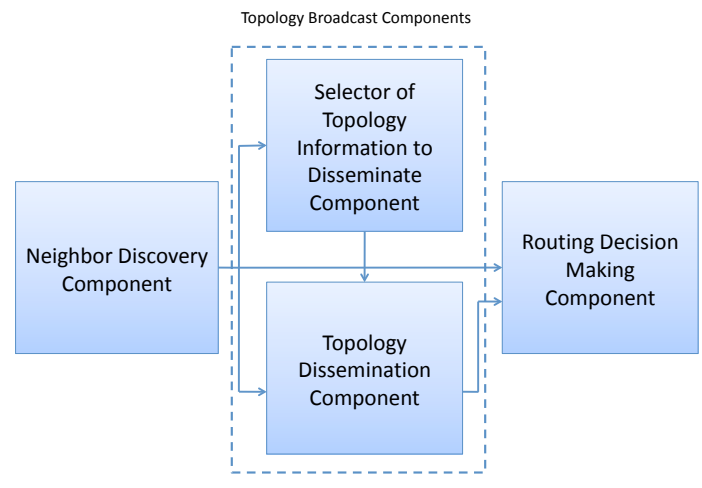

Figure 3: Components of link state Routing Protocols

\section{Selector of Topology Information to Dis- seminate Component}

As the name signifies, the STIDC is responsible for selecting the information that creates a global view for every routing agent. This information could represent coarse details such as a link's ON-OFF state or more precise details such as the interface delay. For instance, in ARPANET the STIDC chooses the average link delays in the network. In OSPF, the cost (also called metric) of an interface is an indication of the overhead required to send packets across a certain interface (i.e. the cost of an interface is inversely proportional to the bandwidth of that interface [1]). The NDC mechanism for both ARPANET and OSPF expose only $\mathcal{N}_{1}(i), i \in V$ and this limits the STIDC to a naive functionality of selecting all information exposed by the NDC. In other words, the STIDC does not have sufficient information to perform local pruning, and hence, there is significant flooding of raw information (Fig. 2). On the contrary, the NDC for OLSR (and many other MANET protocols) exposes $\mathcal{N}_{2}(i)$, and this enables their STIDC to prune the topology information. This pruning guarantees that the shortest path in terms of hop count is preserved in the global view for OLSR. However, for mobile networks it is very natural to associate a cost to the wireless link, based on its stability, capacity or metrics of reliability. In these cases, an OLSR-like STIDC, which is based on the covering condition, is handicapped with respect to meeting the functional requirements.

The STIDC is a significant component for MANET routing protocols because the STIDC serves as the interface between the local and global views of the dynamic graph. While the NDC quickly exposes a local dynamic $\mathcal{N}_{k}(i)[t], i \in V$, to the STIDC, the later has the responsibility of choosing information to create a global view. Ideally, one would expect to flood all the local information. But this results in significant overhead, which consumes the already limited bandwidth of a wireless medium. Instead, if the STIDC can summarize the sufficient information for routing, it can help improve the throughput.

In [9], Wu et al. introduce the concept of local view at every station. This captures the time-varying adjacency at the local neighborhood of every station $i \in V$. We extend this notion to also capture the various metric weights.

Definition The LocalView $w_{i}[t], t \geq 0$, at station $i \in V$ is the subgraph of the dynamic graph $G[t]$ along with the metric weights $c(i, j)[t],(i, j)$ in the subgraph that is exposed at $i$ by its NDC.

For instance, Fig. 4 shows the local view at station $i$ for a NDC mechanism that exposes $\mathcal{N}_{2}(i), i \in V$. We assume that in the neighbor discovery phase, along with the station identifiers, the interface cost $c(i, j)[t]$ is also exchanged. In other words, the LocalView $i[t]$ is composed of $\mathcal{N}_{k}(i)[t]$ along with the metric weights for each link in it. Given this LocalView $i[t]$, the 


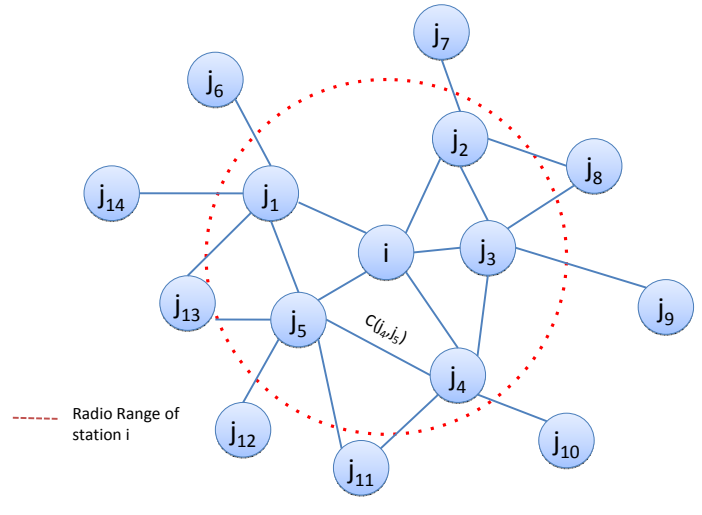

Figure 4: Local view at station $i$

functional requirement of the STIDC is to summarize LocalView $_{i}[t], t \geq 0$, such that the union of this processed information at every $i \in V$ along with the information exposed by the NDC is sufficient for every routing agent to perform a global minimization to compute the optimal route profile.

Definition The GlobalView $i t], t \geq 0$, at station $i \in V$ is the subgraph of the dynamic graph $G[t]$ along with the metric weights $c(i, j)[t],(i, j)$ in this subgraph that is made available at station $i$ by the LocalView $_{i}[t]$, and the broadcast STIDC information.

\subsection{STIDC algorithms}

We now introduce STIDC instances that satisfy the functional description for a set of commonly used routing objective functions. In all the algorithms that follow, every station $i \in V$ runs the STIDC pruning algorithm to summarize its LocalViewi $[t]$. For each station, the algorithm returns a subset of the links incident to that station along with their link costs $\left(\mathcal{L}_{i}\right)$. This information is fed into the TDC to be broadcast across the network. The broadcast subgraph corresponds to $G_{\text {broadcast }}[t]=\cup_{i \in V} \mathcal{L}_{i}[t]$. Then, the corresponding global view at every station $i$ is GlobalView $_{i}[t]=$ LocalView $_{i}[t] \cup G_{\text {broadcast }}[t]$. We show that for a very general class of routing objectives, this GlobalView $[t]$ contains sufficient information for the routing agents to compute the optimal paths.

\subsection{Preserving Shortest Path}

One of the most common routing metrics is the shortest path metric. The length of any path $p$ at time $t$ is given by

$$
l(p)[t]=\sum_{(i, j) \in p} c(i, j)[t]
$$

Then, the shortest path between a source-destination pair $(S, T)$ is

$$
p^{S P}(S, T)[t]=\arg \min _{p \in \mathcal{P}_{S, T}[t]} l(p)[t]
$$

where $\mathcal{P}_{S, T}[t]$ is the set of all paths from $S$ to $T$. The functionality of the STIDC is to preserve the shortest paths in GlobalView $i[t], i \in V$. Running Algorithm 1 at every station $i$ creates a GlobalView $i[t], i \in V$, in the manner described in subsection 6.1.
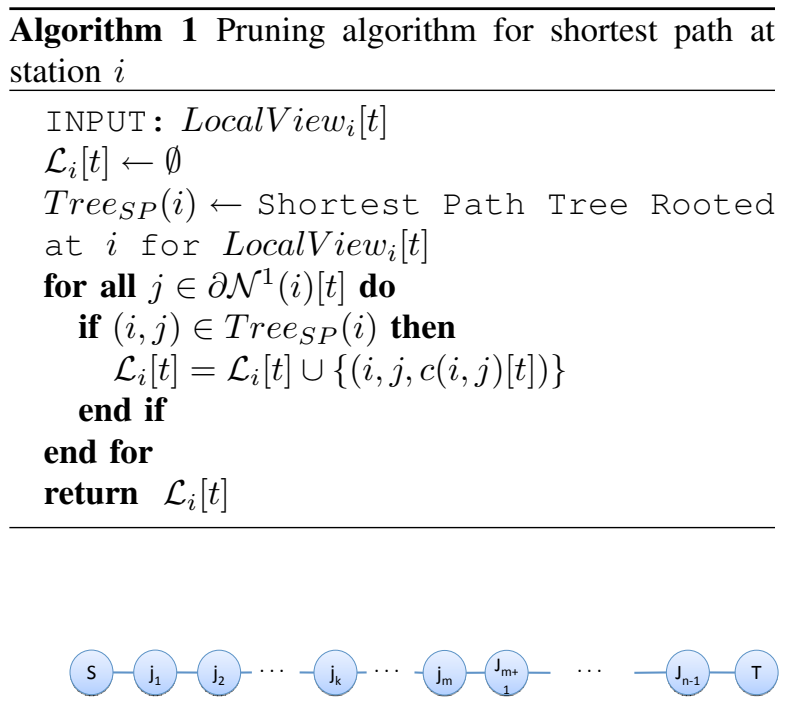

(a) Original Path

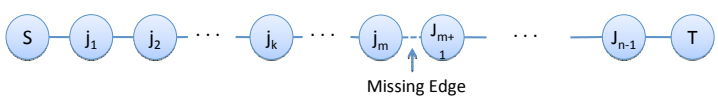

(b) Broken Path

Figure 5: Path $(S \rightarrow T)$

Theorem 6.1: At every station $i \in V$, the GlobalView $w_{i}[t]$ generated by Algorithm 1 preserves all pair source-destination shortest paths in $G[t]$.

Proof: Let us consider any source-destination pair $(S, T)$. Let the shortest path from $S$ to $T$ be $p^{S P}(S, T)[t]=S \rightarrow j_{1} \rightarrow j_{2} \rightarrow \cdots \rightarrow j_{n-1} \rightarrow T$.

This is shown in Fig. 5a. Let us suppose that this shortest path is not preserved in GlobalView ${ }_{S}[t]$. Let us consider the intersection of $p^{S P}(S, T)[t]$ and GlobalView ${ }_{S}[t]$ shown in Fig. 5b. Since the shortest path is not preserved, this corresponds to a broken path. 
Let us choose one missing link $\left(j_{m}, j_{m+1}\right)$.

$\Rightarrow$ Edge $\left(j_{m}, j_{m+1}\right)$ is not a part of the shortest path from $j_{m}$ to $j_{m+1}$. (By Algorithm 1)

$\Rightarrow \exists$ a shortest path $\left(j_{m} \rightarrow j_{l} \rightarrow \cdots \rightarrow j_{m+1}\right)$, where $j_{l} \neq j_{m+1}$ in the LocalView $j_{m}[t]$.

Let us denote by $p^{R}(S, T)$ the path obtained by replacing the edge $\left(j_{m}, j_{m+1}\right)$ in $p^{S P}(S, T)[t]$ with this lesser cost sub-path. Then cost $l\left(p^{S P}(S, T)[t]\right)>$ $l\left(p^{R}(S, T)[t]\right)$. This is a contradiction. So edge $\left(j_{m}, j_{m+1}\right)$ is indeed preserved. We can extend the proof to every missing edge to prove that $p^{S P}(S, T)[t]$ is preserved in GlobalView $i[t]$.

\subsection{Preserving Max-min Paths}

Another routing metric is the bottleneck metric, which is typically used to route traffic through the maximum capacity path. For any path $p$ the bottleneck metric is given by

$$
b(p)[t]=\min _{(i, j) \in p} c(i, j)[t]
$$

Then the max-min path between the source-target pair $(S, T)$ is given by

$$
p^{M M}(S, T)[t]=\arg \max _{p \in \mathcal{P}_{S, T}[t]} b(p)[t]
$$

Algorithm 2 runs at every station $i$ and creates a GlobalView $i[t]$ at $i$.

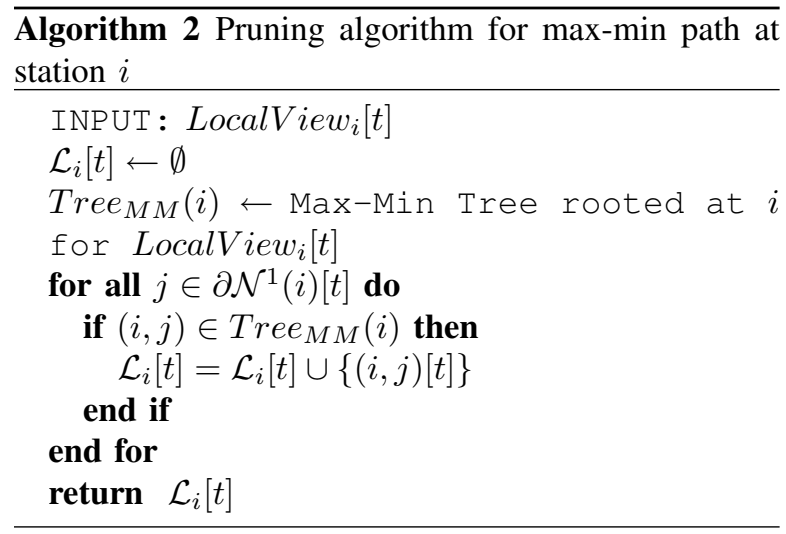

Theorem 6.2: At every station $i \in V$, the GlobalView $_{i}[t]$ generated by Algorithm 2 preserves all pair source-destination max-min paths in $G[t]$.

Proof: Let us consider any source-destination pair $(S, T)$. Let the max-min path from $S$ to $T$ be $p^{M M}(S, T)[t]=S \rightarrow j_{1} \rightarrow j_{2} \rightarrow \cdots \rightarrow j_{n-1} \rightarrow T$. This is shown in Fig. 5a. Let us suppose that this max-min path is not preserved in GlobalView $[t]$. Let us consider the intersection of $p^{M M}(S, T)[t]$ and GlobalView $_{S}[t]$ shown in Fig. 5b. Let us choose one missing link $\left(j_{m}, j_{m+1}\right)$.

$\Rightarrow$ Edge $\left(j_{m}, j_{m+1}\right)$ is not a part of the max-min path from $j_{m}$ to $j_{m+1}$. (By Algorithm 2)

$\Rightarrow \exists$ a max-min path $\left(j_{m} \rightarrow j_{l} \rightarrow \cdots \rightarrow j_{m+1}\right)$, where $j_{l} \neq j_{m+1}$ in the LocalView $j_{m}[t]$.

Let us denote by $p^{R}(S, T)[t]$ the path obtained by replacing the edge $\left(j_{m}, j_{m+1}\right)$ in $p^{M M}(S, T)[t]$ with a better max-min sub-path. Then the bottleneck metric $b\left(p^{M M}(S, T)[t]\right)<b\left(p^{R}(S, T)[t]\right)$. This a contradiction. So edge $\left(j_{m}, j_{m+1}\right)$ is indeed preserved. We can extend the proof to every missing edge to prove that $p^{M M}(S, T)[t]$ is preserved in GlobalView ${ }_{i}[t]$.

\subsection{Preserving K-Shortest Paths}

Another routing objective is the K-shortest paths, which is used for reliability, security and loadbalancing. For any source-destination pair $(S, T)$, the $\mathrm{K}$-shortest paths are the first $\mathrm{K}$ paths of the set $\mathcal{P}_{S, T}[t]$ ranked in increasing path lengths. Again the functionality of the STIDC is to preserve these paths for every source-destination pair. The STIDC runs Algorithm 3 to prune for this set of paths.

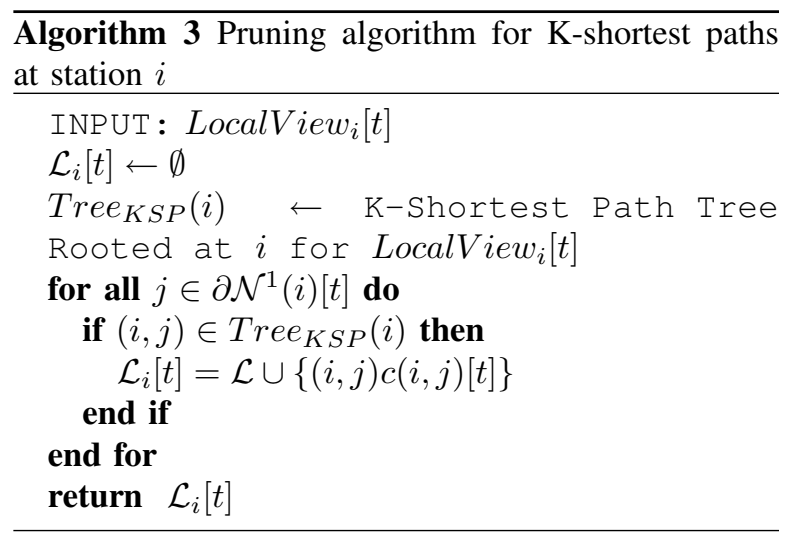

Theorem 6.3: At every station $i \in V$, the GlobalView $_{i}[t]$ generated by Algorithm 3 preserves all $K$-shortest path sets in $G[t]$.

Proof: Let us consider any source-target pair $(S, T)$. Let the K-shortest path set be $\mathcal{P}_{S, T}^{K S P}[t]$. Let us suppose this set of paths is not preserved in GlobalView $_{S}[t]$. Let us consider the intersection of $\mathcal{P}_{S, T}^{K S P}[t]$ with the GlobalView $[t]$. This creates a broken set of paths shown in fig. 6. Let us consider a missing link $\left(j_{m}, j_{m+1}\right)$.

$\Rightarrow$ Edge $\left(j_{m}, j_{m+1}\right)$ is not a part of the $K$-Shortest path set from $j_{m}$ to $j_{m+1}$. (By Algorithm 3)

$\Rightarrow \exists$ a path set $\mathcal{P}_{j_{m}, j_{m+1}}^{K S P}[t]$ in the LocalView ${ }_{i}[t]$ such that none of the paths in the set use the edge $\left(j_{m}, j_{m+1}\right)$. 
Again, we have a better replacement path set between the $(S, T)$ pair using the path set $\mathcal{P}_{S, T}^{K S P}[t]$. We can extend the proof to every missing edge to prove that $\mathcal{P}_{S, T}^{K S P}[t]$ is indeed preserved in GlobalView $\left.i t\right]$.

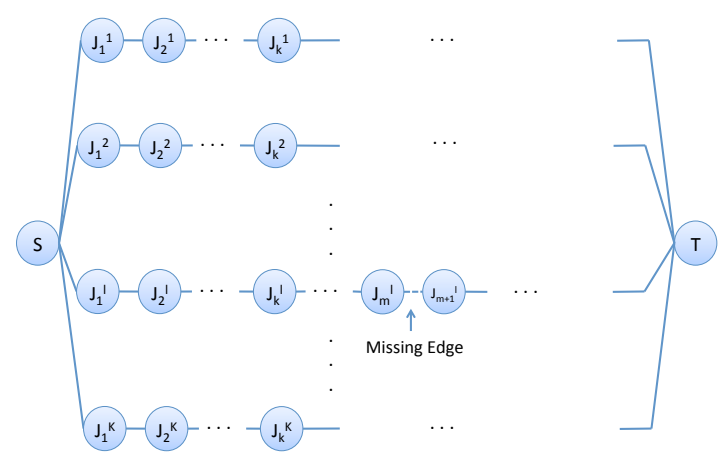

Figure 6: Broken Path Set between $(S, T)$

\section{Generalized Semiring Pruning Methods}

The pruning methods introduced in the previous sections suggest that there is an underlying algebra to these pruning methods. The algorithms suggest that by preserving a property in the local neighborhood, we are able to preserve the property globally. We show that this algebra is a semiring algebra. For a detailed exposition on semirings, we refer the reader to [6], [12], and [13].

A semiring is an algebraic structure $(S, \oplus, \otimes)$ that satisfies the following axioms:

(A1) $(S, \oplus)$ is a commutative semigroup with a neutral element (1)

$$
\begin{aligned}
a \oplus b & =b \oplus a \\
a \oplus(b \oplus c) & =(a \oplus b) \oplus c \\
a \oplus(0) & =a
\end{aligned}
$$

$(A 2)(S, \otimes)$ is a semigroup with a neutral element (1) and (1) as an absorbing element

$$
\begin{aligned}
a \otimes(b \otimes c) & =(a \otimes b) \otimes c \\
a \otimes \mathbb{1} & =a \\
a \otimes \mathbb{( D} & =\text { (D) }
\end{aligned}
$$

$(A 3) \otimes$ distributes over $\oplus$

$$
\begin{aligned}
a \otimes(b \oplus c) & =(a \otimes b) \oplus(a \otimes c) \\
(a \oplus b) \otimes c & =(a \otimes c) \oplus(b \otimes c)
\end{aligned}
$$

It should be noted that the functions that have this semiring structure lend themselves to distributed computation/evaluation by the virtue of the distributivity property (A3). This property of semiring structures has been used in many path problems in graphs [6]. One particularly useful class of semirings for optimization is the ordered semiring class. Here the $\oplus$ is the supremum or infimum operator and $(S, \otimes, \preceq)$ is an ordered semigroup. An ordered semigroup is a semigroup with an order relation which is monotone with respect to $\otimes$. i.e. $a, b, a^{\prime}, b^{\prime} \in S$ we have

$$
a \preceq b \quad \text { and } \quad a^{\prime} \preceq b^{\prime} \quad \Rightarrow \quad a \otimes a^{\prime} \preceq b \otimes b^{\prime}
$$

In this paper, we consider only ordered semirings. Without loss of generality, we assume that the $\oplus$ operator is the infimum operator. In the context of mobile networks with metrics on the links, we associate with every edge $(i, j)$ of the dynamic graph a semiring element $c(i, j)[t] \in S$.

Definition A general semiring path problem on a dynamic graph corresponds to computing

$$
p^{*}(S, T)[t]=\arg \oplus_{p \in \mathcal{P}_{S, T}[t]} \otimes_{(i, j) \in p} c(i, j)[t]
$$

The equivalence of this definition with shortest path, max-min path and k-shortest path problems is well illustrated in [6]. Let us consider an abstract pruning algorithm at every $i \in V$ in Algorithm 4. The procedure Semiring Pruned Tree Rooted at $i$ computes the optimal paths from $i$ to $j \in$ LocalView $_{i}[t]$ based on Equation (1). The algorithm creates a GlobalView $[t], i \in V$, by the procedure illustrated in subsection 6.1.

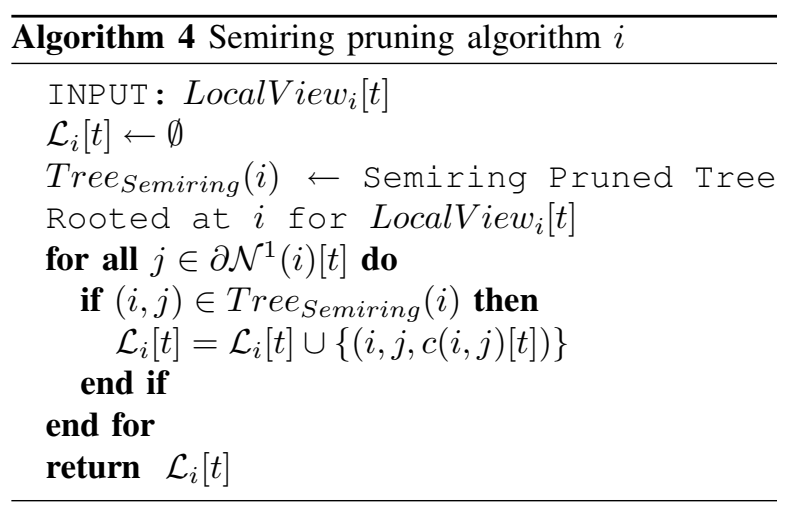

Theorem 7.1: At every station $i \in V$, the GlobalView $w_{i}[t]$ generated by Algorithm 4 preserves all pair optimal paths (optimality with respect to Equation (1)) in $G[t]$.

Proof: Let us consider any source-destination pair $(S, T)$. Let the optimal path from $S$ to $T$ be 
$p^{*}(S, T)[t]=S \rightarrow j_{1} \rightarrow j_{2} \rightarrow \cdots \rightarrow j_{n-1} \rightarrow T$. Let us suppose that this optimal path is not preserved in GlobalView $w_{S}[t]$. Let us consider the intersection of $p^{*}(S, T)$ and GlobalView $w_{S}[t]$. Let us choose one missing link $\left(j_{m}, j_{m+1}\right)$.

$\Rightarrow$ Edge $\left(j_{m}, j_{m+1}\right)$ is not a part of the optimal path from $j_{m}$ to $j_{m+1}$. (By Algorithm 4)

$\Rightarrow \exists$ a optimal path $\left(j_{m} \rightarrow j_{l} \rightarrow \ldots j_{l^{\prime}} \rightarrow j_{m+1}\right)$, where $j_{l} \neq j_{m+1}$ in the LocalView $w_{i}[t]$.

$\Rightarrow c\left(j_{m}, j_{m+1}\right) \succ c\left(j_{m}, j_{l}\right) \otimes \cdots \otimes c\left(j_{l^{\prime}}, j_{m+1}\right)$

$$
\begin{aligned}
\operatorname{Cost}\left(p^{*}(S, T)[t]\right)= & \otimes_{(i, j) \in p^{*}(S, T)[t]} c(i, j)[t] \\
= & c\left(S, j_{1}\right)[t] \otimes c\left(j_{1}, j_{2}\right) \otimes \ldots \\
& \otimes c\left(j_{m}, j_{m+1}\right) \otimes \ldots c\left(j_{n-1}, T\right) \\
\succ & c\left(S, j_{1}\right)[t] \otimes c\left(j_{1}, j_{2}\right) \otimes \cdots \otimes \\
& c\left(j_{m}, j_{l}\right) \otimes \cdots \otimes c\left(j_{l^{\prime}}, j_{m+1}\right) \otimes \\
& \cdots \otimes c\left(j_{n-1}, T\right)(B y A 3) .
\end{aligned}
$$

This means there is a better path from $S$ to $T$. This is a contradiction. Edge $\left(j_{m}, j_{m+1}\right)$ is indeed preserved. We can extend the proof to every missing edge to prove that $p^{*}(S, T)$ is preserved in GlobalView $w_{i}[t]$.

This generalization using semiring distribution is not necessarily limited to routing objectives. The same architectural abstractions can be extended to other applications such as sensor fusion, estimation and tracking (many of these algorithms are message passing algorithms which can be abstracted as semirings).

\section{Conclusion}

In this paper, we defined the functional requirements of STIDC. We detailed the importance of the STIDC pruning for routing in MANETs. We then presented instances of the STIDC, which aid the routing agents to correctly configure their routing tables. We showed that these instances can preserve important properties such as shortest paths, min-max paths and K-shortest paths by local pruning. We also generalized this property and showed that it is a special case of the semiring distribution property.

\section{Acknowledgment}

This material is based upon work supported by the Communications and Networks Consortium sponsored by the U.S. Army Research Laboratory under the Collaborative Technology Alliance Program, Cooperative Agreement DAAD19-01-2-0011 and the MURI Award Agreement W911-NF-0710287 from the Army Research Office.

\section{References}

[1] Ospf design guide. www.cisco.com/en/US/tech/tk365/ technologies_white_paper09186a0080094e9e.shtml. Last accessed 05/30/2009.

[2] Qayyum A., Viennot L., and Laouiti A. Multipoint relaying for flooding broadcast messages in mobile wireless networks. In Proceedings of the 35th Annual Hawaii International Conference on System Sciences (HICSS'02), volume 9, page 298, 2002.

[3] Ben Khedher D., Glitho R., and Dssouli R. A novel overlay-based failure detection architecture for manet applications. In IEEE International Conference on Networks, pages 130-135, 2007.

[4] Johnson D.B. Routing in ad hoc networks of mobile hosts. In IEEE Workshop on Mobile Computing Systems and Applications, pages 158-163, 1994.

[5] Bertsekas D.P. and Gallager R. Data Networks. Prentice Hall, 1992.

[6] Rote G. Path problems in graphs. In Computing Supplementum, volume 7, pages 155-189, 1990.

[7] McQuillan J., Richer I., and Rosen E. The new routing algorithm for the arpanet. IEEE Transactions of Communication, 28:711- 719, 1980.

[8] McQuillan J., Richer I., and Rosen E. An overview of the new routing algorithm for the arpanet. In ACM SIGCOMM Computer Communication Review, volume 25, pages 54-60, 1995.

[9] Wu J. and Dai F. A generic distirbuted broadcast scheme in ad hoc wireless networks. IEEE Transactions of Computers, 53(10):1343-1354, 2004.

[10] Baras J.S., Tabatabaee V., Purkayastha P., and Somasundaram K. Component based performance modeling of the wireless routing protocols. In IEEE ICC Ad Hoc and Sensor Networking Symposium, pages 1-6, June 2009.

[11] Jacquet P., Laouiti A., Minet P., and Viennot L. Performance of multipoint relaying in ad hoc mobile routing protocols. Springer, February 2004.

[12] McEliece R.J. and Aji S.M. The generalized distributive law. IEEE Transactions on Information Theory, 46(2):325-343, 2000.

[13] Verdu S. and Poor V. Abstract dynamic programming models under commutativiy conditions. SIAM Journal on Control and Optimization, 25(4):990-1006, 1987.

[14] Clausen T. and Jacquet P. Optimized link state routing protocol (olsr). RFC, Oct 2003. 